\title{
The Asymptotic Behavior of the Solutions of $(\Delta+\lambda) u=0$ in a Domain with the Unbounded Boundary
}

\author{
By \\ Takao TAYoshi*
}

\section{1. 耳ntroduction}

We shall consider the equation

$$
(\Delta+\lambda) u=0
$$

in an unbounded domain $\Omega$ in the Euclidean $n$-space $E^{n}(n \geqq 2)$, with the boundary condition

$$
\left.u\right|_{\Gamma}=0
$$

where $\Gamma$ is the boundary of $\Omega$, and $\lambda$ is a positive constant. Let $\Omega(L)=$ $\Omega_{\cap}\left\{\left(x_{1}, \ldots, x_{n}\right) \in E^{n}: x_{1}>L\right\}$. We shall assume that $\Gamma$ is smooth $\left(C^{1}\right)$, and that there are positive numbers $C, N$ and $l(l \leqq 1)$ such that the following (1.3) and (1.4) hold for at least one of the connected components of $\Omega(N)$, say $\Omega_{1}(N)$.

$$
\begin{gathered}
\left.\Omega_{1}(N) \subset\left\{x_{1}, \cdots, x_{n}\right) \in E^{n}:\left(x_{2}^{2}+\cdots+x_{n}^{2}\right)^{\frac{1}{2}}<C x_{1}^{l}\right\} \\
\mathbf{n}(p) \cdot \mathbf{a}(p) \leqq 0 \quad \text { for } p \in \Gamma_{\cap} \partial \Omega_{1}(N)
\end{gathered}
$$

where $\mathbf{n}(p)$ is the outer unit normal to $\Gamma$ at $p=\left(x_{1}, \ldots, x_{n}\right)$ and $\mathbf{a}(p)$ is the vector $\mathbf{a}(p)=\left(x_{1}, l x_{2}, \ldots, l x_{n}\right)$. Our purpose in this paper is to prove the following.

Received July 3, 1972.

Communicated by S. Matsuura.

* Department of Mathematics, Osaka Institute of Technology, Omiya 1, Asahi-ku, Osaka 535, Japan. 
Theorem $\mathbb{1}$. 1 . Let $\Omega$ and $\lambda$ be as above. If $u$ is a non-trivial solution of (1.1) and (1.2), then

$$
\lim _{t \rightarrow \infty} t^{\varepsilon} \int_{P_{t}}\left(u^{2}+|\nabla u|^{2}\right) d S=\infty
$$

for any $\varepsilon>0$, where $P_{t}$ is the section of $\Omega_{1}(N)$ by the hyperplane $x_{1}=t$.

If $\Omega$ lies in the half-space $x_{1}>1$, and (1.4) holds on the whole of $\Gamma$ with $l=0 . \quad(1.5)$ is a part of the well known results by Rellich [1]. Jones [2] (Theorem 9) has treated the problem in the case of $l=$ 1. We can find in Agmon [3] (Theorem 11) an extension of Jones' result, and, when $l=1$, our Theorem 1.1 is also included in Agmon's theorem. So the proof of Theorem 1.1 must be carried out for $0<l<1$, and it will be done in the framework developed by Roze [4] and Eidus [5].

In $\S 2$, introducing a curvilinear coordinate system for the convenience of calculations, we shall give some preliminary lemmas. In $\S 3$, it will be shown that a solution which does not satisfy (1.5) decreases, in a sense, like an exponential function in $\Omega_{1}(N)$, and in $\S 4$, it will turn out that such solution is the trivial solution.

In consequence of Theorem 1.1 it is easy to see that the self-adjoint realization of $-\Delta$ in $L^{2}(\Omega)$ with the Dirichlet boundary condition has no positive point eigenvalues. A short remark on the spectrum will be given in the final $\S 5$.

\section{Preliminaries}

In the sequel the conditions of the Theorem 1.1 are always assumed. Let us introduce a curvilinear coordinate system $\left(X_{1}, \ldots, X_{n}\right)$ in $E_{+}^{n}=\left\{\left(x_{1}\right.\right.$, $\left.\left.\cdots, x_{n}\right): x_{1}>0\right\}$ as follows;

$$
\begin{cases}X_{1}=\left\{x_{1}^{2}+l\left(x_{2}^{2}+\cdots+x_{n}^{2}\right)\right\}^{\frac{1}{2}} & \left(X_{1}>0\right), \\ X_{2}=\tan ^{-1}\left\{\left(x_{2}^{2}+\cdots+x_{n}^{2}\right)^{\frac{1}{2}} / x_{1}^{l}\right\} & \left(0 \leqq X_{2}<\frac{\pi}{2}\right),\end{cases}
$$

and $X_{3}, \ldots, X_{n}$ are the parameters which are suitably chosen on the sphere $S^{n-2}=\left\{\left(x_{2}, \cdots, x_{n}\right): x_{2}^{2}+\cdots+x_{n}^{2}=1\right\}$. (For example, we may put $x_{2}=$ 
$\cos X_{3}, x_{3}=\sin X_{3} \cos X_{4}, \cdots, x_{n-1}=\sin X_{3} \ldots \sin X_{n-1} \cos X_{n}, x_{n}=\sin X_{3} \cdots \sin$ $X_{n}, 0 \leqq X_{i} \leqq \pi$ for $3 \leqq i \leqq n-1$ and $0 \leqq X_{n} \leqq 2 \pi$.) Let $(d s)^{2}=\sum_{i=1}^{n}\left(d x_{i}\right)^{2}$ $=\sum_{i . j=1}^{n} g_{i j} d X_{i} d X_{j}$ be the ordinary Euclidean metric. Then we can see $g_{11}=X_{1}^{2} /\left\{X_{1}^{2}+\left(l^{2}-l\right) r^{2}\right\}, g_{12}=g_{21}=0, g_{22}=x_{1}^{2 l+2} \sec ^{4} X_{2} /\left\{X_{1}^{2}+\left(l^{2}-l\right) r^{2}\right\}$ and $g_{i j}=r^{2} \tilde{g}_{i j}$ for $i, j \geqq 3$, where $r=\left(x_{2}^{2}+\cdots+x_{n}^{2}\right)^{\frac{1}{2}}$ and $\sum_{i . j=3}^{n} \tilde{g}_{i j} d X_{i} d X_{j}$ is the metric on the sphere $S^{n-2}$ induced from $E^{n-1}=\left\{\left(x_{2}, \cdots, x_{n}\right)\right\}$. Put $\left(g^{i j}\right)=\left(g_{i j}\right)^{-1}$ and $G=\operatorname{det}\left(g_{i j}\right)$. Then

$$
\left\{\begin{aligned}
\Delta f & \equiv \sum_{i=1}^{n} \partial^{2} f / \partial x_{i}^{2}=(1 / \sqrt{G}) \sum_{i \cdot j=1}^{n}\left(g^{i j} \sqrt{G} f_{X_{\jmath}}\right)_{X_{i}} \\
|\nabla f|^{2} & \equiv \sum_{i=1}^{n}\left|\partial f / \partial x_{i}\right|^{2}=\sum_{i . j=1}^{n} g^{i j} f_{X_{\imath}} \bar{f}_{X_{j}}
\end{aligned}\right.
$$

for a smooth function $f$, where $f_{X_{i}}=\partial f / \partial X_{j}$.

Now we give some lemmas specifying the asymptotic properties of $g^{i j}$ and $G$, which will play important roles in the following sections.

Lemma 2.1. $g^{11} \rightarrow 1, X_{1} g_{X_{1}}^{11} / g^{11} \rightarrow 0, X_{1} g_{X_{1}}^{i j} / g^{i j} \rightarrow 2 l(i=j=2$ or $i, j \geqq 3)$ and $X_{1} G_{X_{1}} / G \rightarrow 2(n-1) l$ when $X_{1} \rightarrow \infty$. These convergences are uniform in $X_{2} \in[0, \theta\rfloor$ for any $\theta<\frac{\pi}{2}$.

Remark. Because of the condition (1.3), there is a number $\theta<\frac{\pi}{2}$, such that $x_{2}<\theta$ for any point in $\Omega_{1}(N)$.

Proof of Lemma 2.1. In the case of $l=1$, the proof is easy. If $0<l<1$, then $r / X_{1} \rightarrow 0\left(X_{1} \rightarrow \infty\right)$ uniformly when $X_{2}$ varies in $[0, \theta]$, because, $r=x_{1}^{l} \tan X_{2}$ and $x_{1} \leqq X_{1}$. From this $g^{11 \rightarrow 1}$ is obvious since $g^{11}=\left\{X_{1}^{2}+\left(l^{2}-l\right) r^{2}\right\} / X_{1}^{2}$. The other convergences can be proved also easily by straightforward calculations if we use the facts that $x_{X_{1}}=x_{1} X_{1}$ $/\left\{X_{1}^{2}+\left(l^{2}-l\right) r^{2}\right\}, r_{X_{1}}=l r X_{1} /\left\{X_{1}^{2}+\left(l^{2}-l\right) r^{2}\right\}, G=g_{11} g_{22} r^{2(n-2)} \operatorname{det}\left(\tilde{g}_{i j}\right)$, and $\tilde{g}_{i j}$ are independent of $X_{1}$ and $X_{2}$.

Q.E.D.

Lemma 2.2. For any real $\delta$, we have $X_{1}^{1-\delta}\left(\sqrt{G} X_{1}^{\delta}\right) / \sqrt{G} \rightarrow \delta+(n-$ 1) $l, X_{1}^{1-\delta}\left(g^{11} \sqrt{G} X_{1}^{\delta}\right)_{X_{1}} / \sqrt{G} \rightarrow \delta+(n-1) l$, and $X_{1}^{1-\delta}\left(g^{i j} \sqrt{G} X_{1}^{\delta}\right)_{X_{1}} / g^{i j} \sqrt{G} \rightarrow$ $\delta+(n-3) l$ for $i=j=2$ or $i, j \geqq 3$, when $X_{1} \rightarrow \infty$. These convergences are 
uniform in $X_{2} \in[0, \theta]$ for any $\theta<\frac{\pi}{2}$.

Proof. Lemma 2.1. and direct calculations lead us to this lemma.

Q.E.D.

Let $\Omega_{A B}, \Omega_{A}$ and $S_{A}$ be the subsets of $\Omega_{1}(N)$ characterized by $A<X_{1}$ $<B, A<X_{1}<\infty$ and $X_{1}=A$ respectively, and put $\Gamma_{A B}=\partial \Omega_{A B}-\left(S_{A} \cup S_{B}\right)$ (the 'side' of $\Omega_{A B}$ ). If $u$ is a solution of (1.1) and (1.2), then $v=X^{m} u$ $(m \geqq 0)$ satisfies

$$
\Delta V-\frac{2 m}{X_{1}} g^{11} V_{X_{1}}+(M+\lambda) V=0 \quad\left(\text { in } \Omega_{A}\right)
$$

and

$$
\left.V\right|_{\Gamma_{A}}=0
$$

for $A>\inf _{\Omega_{1}(N)} X_{1} \equiv N_{0}$, where $M=\left(m^{2}+m\right) g^{11} / X_{1}^{2}-m\left(g^{11} \sqrt{G}\right)_{X_{1}} / X_{1} \sqrt{G}$.

Lemma 2.3. $X_{1}^{2} M-g^{11} m^{2} \rightarrow m(1-(n-1) l)$ uniformly when $X_{1} \rightarrow \infty$ in $\Omega_{1}(N)$, and there exist positive constants $C_{1}$ and $N_{1}$ which are independent of $m \geqq 0$ such that the inequalities $M \geqq 0$ and $X M_{X_{1}}+2\left(m / X_{1}\right)^{2} \leqq$ $m C_{1} / X_{1}^{2}$ hold in $\Omega_{A}$ for $A>N_{1}$.

Proof. It is easy to prove that $g \frac{11}{X_{1} X_{1}}=o\left(1 / X_{1}^{2}\right)$ and $G_{X_{1} X_{1}}=O\left(1 / X_{1}^{2}\right)$ as $X_{1} \rightarrow \infty$ in $\Omega_{1}(N)$. Using these facts and Lemmas $2.1-2$, we have the lemma.

Q.E.D.

The next two lemmas are concerned with the solutions of (2.3) and (2.4).

Lemma 2.4. Let $v$ be a real valued solution of (2.3) and (2.4). Then

$$
\begin{aligned}
\int_{\Omega_{A B}} \psi|\nabla v|^{2} d \Omega & =\left\{\int_{S_{B}}-\int_{S_{A}}\right\} \sqrt{g^{11}} \psi v v_{X_{1}} d S \\
- & \int_{\Omega_{A B}}\left(\psi^{\prime}+\frac{2 m \psi}{X_{1}}\right) g^{11} v v_{X_{1}} d \Omega+\int_{\Omega_{A B}} \psi(M+\lambda) v^{2} d \Omega
\end{aligned}
$$


where $B>A>N_{0}$, and $\psi$ is smooth and depends only on $X_{1}$.

Proof. Multiply (2.3) by $\phi v$ and integrate over $\Omega_{A B}$. Using (2.4) we have (2.5).

Q.E.D.

If we put $m=0$ in (2.5),

$$
\begin{aligned}
\int_{\Omega_{A B}} \psi|\nabla u|^{2} d \Omega & =\left\{\int_{S_{B}}-\int_{S_{A}}\right\} \sqrt{g^{11}} \psi u u_{X_{1}} d S \\
& -\int_{\Omega_{A B}} g^{11} \psi^{\prime} u u_{X_{1}} d \Omega+\lambda \int_{\Omega_{A B}} \psi u^{2} d \Omega .
\end{aligned}
$$

Lemma 2.5. Let $v$ be a real valued solution of (2.3) and (2.4). For any $\delta>0, \eta>0$ and $m \geqq 0$, we can find a real $N_{2}=N_{2}(\delta, \eta)$ which is independent of $m$ such that the inequality

$$
\begin{aligned}
& \left\{\int_{S_{B}}-\int_{S_{A}}\right\} X_{1}^{\delta}\left\{g^{11} v_{X_{1}}^{2}-\frac{|\nabla v|^{2}}{2}+\frac{(M+\lambda)}{2} v^{2}\right\} \sqrt{g_{11}} d S \\
& -(2 m+\delta-l) \int_{\Omega_{A B}} X^{\delta-1} g^{11} v_{X}^{2} d \Omega \\
& +\frac{1}{2} \int_{\Omega_{A B}} X_{1}^{\delta-1}\left[(\delta+(n-3) l+\eta)|\nabla v|^{2}\right. \\
& \left.-\left\{(\delta+(n-1) l-\eta)(M+\lambda)+X_{1} M_{X_{1}}\right\} v^{2}\right] d \Omega \\
& \geqq 0
\end{aligned}
$$

holds for $B>A>N_{2}$.

Proof. We multiply (2.3) by $X_{1}^{\delta} v_{X_{1}}$ and integrate over $\Omega_{A B}\left(A>N_{0}\right)$. Integrating by parts, we have

$$
\begin{aligned}
& \left\{\int_{S_{B}}-\int_{S_{A}}\right\} X_{1}^{\delta}\left\{g^{11} v_{X_{1}}^{2}-\frac{|\nabla v|^{2}}{2}+\frac{(M+\lambda)}{2} v^{2}\right\} \sqrt{g_{11}} d S \\
& -2 m \int_{\Omega_{A B}} X_{1}^{\delta-1} g^{11} v_{X_{1}}^{2} d \Omega-\int_{A B}\left\{\delta X_{1}^{\delta-1} g^{11 \sqrt{G}}\right. \\
& \left.-\frac{1}{2}\left(g^{11} \sqrt{G} X_{1}^{\delta}\right)_{X_{1}}\right\} v_{X_{1}}^{2} \frac{d \Omega}{\sqrt{G}}+\frac{1}{2} \int_{\Omega_{A B}}\left[\sum_{i . j \geqq 2}\left(g^{i j} \sqrt{G} X_{1}^{\delta}\right)_{X_{1}} v_{X_{i}} v_{X_{j}}\right.
\end{aligned}
$$




$$
\begin{aligned}
& \left.-\left\{(M+\lambda) \sqrt{G} X_{1}^{\delta}\right\}_{X_{1}} v^{2}\right] \frac{d \Omega}{\sqrt{G}} \\
& =-\frac{1}{2} \int_{\Gamma_{A B}} X_{1}^{\delta}|\nabla v|^{2} \sqrt{g_{11}}\left(\boldsymbol{n} \cdot \boldsymbol{X}_{1}\right) d S
\end{aligned}
$$

where $\boldsymbol{X}_{1}$ is the vector $\boldsymbol{X}_{1}=\frac{a}{|a|}=\left(x_{1}, l x_{2}, \cdots, l x_{n}\right) /\left(x_{1}^{2}+l^{2} x_{2}^{2}+\cdots+l^{2} x_{n}^{2}\right)^{\frac{1}{2}}$. Here we have used the fact that $v_{X_{1}}(\nabla v \cdot \boldsymbol{n})=\sqrt{ } g_{11}|\nabla v|^{2}\left(\mathbb{X}_{1} \cdot \boldsymbol{n}\right)$ on $\Gamma_{A B}$, which follows from the boundary condition (2.4). In view of the condition (1.4), the right side of (2.8) is non-negative. In consequence of Lemma 2.2 , for any $\eta>0$, we can take $N_{2}^{\prime}(\delta, \eta)$ such that the inequalites

$$
\begin{aligned}
& \delta g^{11} X_{1}^{\delta-1}-\frac{1}{2}\left(g^{11} \sqrt{G} X_{1}^{\delta}\right)_{X_{1}} / \sqrt{G} \geqq \frac{1}{2}(\delta-(n-1) l-\eta) g^{11} X_{1}^{\delta-1} \\
& \left(g \sqrt{G} X_{1}^{\delta}\right)_{X_{1}} / \sqrt{G} \leqq(\delta+(n-3) l+\eta) g^{i j} X_{1}^{\delta-1}(i, j \geqq 2) \\
& \left(\sqrt{G} X_{1}^{\delta}\right)_{X_{1}} \geqq(\delta+(n-1) l-\eta) X_{1}^{\delta-1}
\end{aligned}
$$

hold if $X_{1}>N_{2}^{\prime}(\delta, \eta)$. Thus we have the inequality (2.7) for $B>A>$ $N_{2}(\delta, \eta)=\max \left(N_{0}, N_{1}, N_{2}^{\prime}(\delta, \eta)\right)$ by Lemma 2.3. $\quad$ Q.E.D.

\section{On a Solution Which Does Not Satisfy (1.5)}

In this and following sections, we use the abbreviations $X, f_{X}$ and $\gamma$ which stand for $X_{1}, f_{X_{1}}=\partial f / \partial X_{1}$ and $g^{11}$ respectively.

Lemma 3.1. Let $u$ be a solution of (1.1) and (1.2). If

$$
\lim _{t \rightarrow \infty} \inf ^{\delta} \int_{S_{t}}\left(|u|^{2}+|\nabla u|^{2}\right) d S=0
$$

for some $\delta>0$, then

$$
\int_{\Omega_{1}(N)} X^{m}\left(|u|^{2}+|\nabla u|^{2}\right) d \Omega<\infty
$$

for any $m \geqq 0$.

Proof. We may assume that $u$ is real valued. If we put $m=0$ in Lemma 2.5, we have 


$$
\begin{aligned}
& \left\{\int_{S_{B}}-\int_{S_{B}}\right\} X^{\delta}\left\{\gamma u_{X}^{2}-\frac{|\nabla u|^{2}}{2}+\lambda u^{2}\right\} \frac{d S}{\sqrt{\gamma}} \geqq \\
& (\delta-l) \int_{\Omega_{A B}} X^{\delta-1} \ell u_{X}^{2} d \Omega-\frac{1}{2} \int_{\Omega_{A B}} X^{\delta-1}\left\{(\delta+(n-3) l+\eta)|\nabla u|^{2}-\right. \\
& \left.\lambda(\delta+(n-1) l-\eta) u^{2}\right\} d \Omega,
\end{aligned}
$$

for $B>A>N_{2}(\delta, \eta)$. On the other hand, taking $X^{\delta-1}$ as $\psi$ in (2.6), we see

$$
\begin{aligned}
& \left\{\int_{S_{B}}-\int_{S_{A}}\right\} X^{\delta-1} u u_{X} \sqrt{\gamma d} S \\
& =\int_{\Omega_{A B}} X^{\delta-1}\left(|\nabla u|^{2}-\lambda u^{2}\right) d \Omega+\int_{\Omega_{A B}} \gamma(\delta-1) X^{\delta-2} u u_{X} d \Omega .
\end{aligned}
$$

From (3.3) and (3.4), we have, for $A>N_{2}$,

$$
\begin{aligned}
& \left\{\int_{S_{B}}-\int_{S_{A}}\right\} X^{\delta}\left\{\gamma u_{X}^{2}-\frac{|\nabla u|^{2}}{2}+\lambda u^{2}\right\} \frac{d S}{\sqrt{\gamma}} \\
& +\frac{(n-1)}{2} l\left\{\int_{S_{B}}-\int_{S_{A}}\right\} X^{\delta-1} u u_{X} \sqrt{\gamma} d S \geqq(\delta-l) \int_{\Omega_{A B}} X^{\delta-1} \gamma u_{X}^{2} d \Omega \\
& -\frac{1}{2} \int_{\Omega_{A B}} X^{\delta-1}\left\{(\delta-2 l+\eta)|\nabla u|^{2}-\lambda(\delta-\eta) u^{2}\right\} d \Omega \\
& +\frac{(n-1)}{2}(\delta-1) l \int_{\Omega_{A B}} \gamma X^{\delta-2} u u_{X} d \Omega \\
& =\frac{1}{2} \int_{\Omega_{A B}} X^{\delta-1}\left\{(\delta-2 \eta)|\nabla u|^{2}+\lambda(\delta-2 \eta) u^{2}\right\} d \Omega \\
& +\left(l-\delta+\frac{\eta}{2}\right) \int_{\Omega_{A B}} X^{\delta-1}|\nabla u|^{2} d \Omega+(\delta-l) \int_{\Omega_{A B}} X^{\delta-1} \gamma u_{X}^{2} d \Omega \\
& +\frac{\eta \lambda}{2} \int_{\Omega_{A B}} X^{\delta-1} u^{2} d \Omega+\frac{(n-1)(\delta-1) l}{2} \int_{\Omega_{A B}} \gamma X^{\delta-2} u u_{X} d \Omega .
\end{aligned}
$$

Without loss of generality, we may assume $\delta<l$. So we can consider that

$$
\left|(\delta-l) \int_{\Omega_{A B}} X^{\delta-1} \gamma u_{X}^{2} d \Omega\right| \leqq(l-\delta) \int_{\Omega_{A B}} X^{\delta-1}|\nabla u|^{2} d \Omega .
$$

Moreover, $\left|(\delta-1)(n-1) u u_{X} / X\right| \leqq \eta\left(\gamma u_{X}^{2}+\lambda u^{2}\right)$ for sufficiently large $X$, 
say $X>N_{3}(\eta, \lambda)$. Thus, passing to the limit for $B \rightarrow \infty$, it follows from (3.5) that

$$
\begin{aligned}
& \int_{S_{A}} X^{\delta}\left(|\nabla u|^{2}-\lambda u^{2}\right) \frac{d S}{\sqrt{\gamma}}-(n-1) l \int_{S_{A}} X^{\delta-1} u u_{X} \sqrt{\gamma} d S \\
& \geqq \frac{\delta}{2} \int X^{\delta-1}\left(|\nabla u|^{2}+\lambda u^{2}\right) d \Omega
\end{aligned}
$$

for $A>N_{4}(\delta, \lambda) \equiv \max \left\{N_{2}\left(\delta, \frac{\delta}{4}\right), N_{3}\left(\frac{\delta}{4}, \lambda\right)\right\}$.

We integrate (3.6) with respect to $A$ from $\xi_{0}$ to $\xi_{1}\left(\xi_{1}>\xi_{0}>N_{4}\right)$. Using $\left|u u_{X}\right|<\left(u^{2}+|\nabla u|^{2}\right) / 2$ and (2.6) in which we replace $\psi$ by $X^{\delta}$, we have

$$
\begin{aligned}
& \frac{\delta}{2} \int_{\xi_{0}}^{\xi_{1}} d \xi \int_{\Omega_{\xi}} X^{\delta-1}\left\{|\nabla u|^{2}+\lambda u^{2}\right\} d \Omega \leqq C_{2} \int_{\Omega_{\xi_{0} \xi_{1}}} X^{\delta-1}\left(|\nabla u|^{2}+u^{2}\right) d \Omega \\
& \quad+\left\{\int_{S_{\xi_{1}}}-\int_{S_{\xi_{0}}}\right\} X^{\delta} u u_{X} \sqrt{\gamma} d S,
\end{aligned}
$$

where $C_{2}=C_{2}(\delta)$ is some positive constant which is independent of $\xi_{0}$ and $\xi_{1}$. By (3.1) and

$$
\begin{aligned}
& \int_{\xi_{0}}^{\xi_{1}} d \xi \int_{\Omega_{\xi}} X^{\delta-1}\left(|\nabla u|^{2}+\lambda u^{2}\right) d \Omega \\
& =\int_{\Omega_{\xi_{0} \xi_{1}}}\left(X-\xi_{0}\right) X^{\delta-1}\left(|\nabla u|^{2}+\lambda u^{2}\right) d \Omega+\left(\xi_{1}-\xi_{0}\right) \int_{\Omega_{\xi_{1}}} X_{1}^{\delta-1}\left(|\nabla u|^{2}+\lambda u^{2}\right) d \Omega
\end{aligned}
$$

(3.7) implies

$$
\begin{aligned}
\frac{\delta}{2} \int_{\Omega_{\xi_{0}}}\left(X-\xi_{0}\right) X^{\delta-1}\left(|\nabla u|^{2}+\lambda u^{2}\right) d \Omega & \leqq C_{2} \int_{\Omega_{\xi_{0}}} X^{\delta-1}\left(|\nabla u|^{2}+u^{2}\right) d \Omega \\
& -\int_{S_{\xi_{0}}} X^{\delta} u u_{X} \sqrt{\gamma} d S
\end{aligned}
$$

$\left(\xi_{0}>N_{4}\right)$. Integrating this inequality with respect to $\xi_{0}$ from $\xi_{1}$ to $\infty$ $\left(\xi_{1}>N_{4}\right)$, we find

$$
\int_{\Omega_{\xi_{1}}}\left(X-\xi_{1}\right)^{2} X^{\delta-1}\left(|\nabla u|^{2}+\lambda u^{2}\right) d \Omega \leqq C_{3} \int_{\Omega_{\xi_{1}}} X^{\delta}\left(|\nabla u|^{2}+u^{2}\right) d \Omega
$$


where $C_{3}$ does not depend on $\xi_{1}$. Repeating this process, we arrive at (3.2).

Q.E.D.

Lemma 3.2. Under the assumption of Lemma 3.1,

$$
\lim _{t \rightarrow \infty} e^{2 \alpha t} \int_{S_{t}}|u|^{2} d S=0
$$

for $\alpha<\sqrt{\lambda l /(1-l)}$. If $l=1, \alpha$ may be taken arbitrarily.

Proof. We may assume that $u$ is real valued. Put $v=X^{m} u$. In Lemma 2.5, we replace $\delta$ by $l$ and let $B \rightarrow \infty$. Then, by Lemma 3.1, we have

$$
\begin{aligned}
& -\int_{S_{A}} X^{l}\left(\gamma v_{X}^{2}-\frac{|\nabla v|^{2}}{2}+\frac{M+\lambda}{2} v^{2}\right) \frac{d S}{\sqrt{\gamma}}-2 m \int_{\Omega_{A}} X^{l-1} \gamma v_{X}^{2} d \Omega \\
& +\frac{1}{2} \int_{\Omega_{A}} X^{l-1}\left[\{(n-2) l+\eta\}|\nabla v|^{2}-\left\{(n l-\eta)(M+\lambda)+X M_{X}\right\} v^{2}\right] d \Omega \\
& \geqq 0
\end{aligned}
$$

for $A>N_{2}(l, \eta)$. On the other hand, taking $X^{l-1}$ as $\psi$ in (2.5) we see

$$
\begin{aligned}
& \int_{\Omega_{A}} X^{l-1}|\nabla v|^{2} d \Omega=-\int_{S_{A}} X^{l-1} \sqrt{\gamma} v v_{X} d S \\
& -\int_{\Omega_{A}} X^{l-2}\{(l-1)+2 m\} \gamma v v_{X} d \Omega+\int_{\Omega_{A}} X^{l-1}(M+\lambda) v^{2} d \Omega .
\end{aligned}
$$

From (3.9) and (3.10), we have

$$
\begin{aligned}
& \int_{S_{A}} X^{l}\left\{\gamma v_{X}^{2}-\frac{|\nabla v|^{2}}{2}+\frac{1}{2}(M+\lambda) v^{2}\right\} \frac{d S}{\sqrt{\gamma}} \\
& +\frac{(n-2) l+\eta}{2} \int_{S_{A}} X^{l-1} \sqrt{\gamma} v v_{X} d S+2 m \int_{\Omega_{A}} X^{l-1} \gamma v_{X}^{2} d \Omega \\
& \left.+\frac{1}{2}(l-1+2 m)\{(n-2) l+\eta)\right\} \int_{S_{A}} X^{l-2} \gamma v v_{X} d \Omega \\
& +\frac{1}{2} \int_{\Omega_{A}} X^{l-1}\left\{2(l-\eta)(M+\lambda)+X M_{X}\right\} v^{2} d \Omega \leqq 0
\end{aligned}
$$


for $A>N_{2}$. Note that the fourth term of (3.11) is estimated as follows;

$$
\begin{aligned}
& \left|\frac{1}{2}(l-1+2 m)\{(n-2) l+\eta\} \int_{\Omega_{A}} \ldots d \Omega\right| \leqq 2 m \int_{\Omega_{A}} X^{l-1} \gamma v_{X}^{2} d \Omega \\
& +\frac{C_{4}}{2} m \int_{\Omega_{A}} X^{l-3} v^{2} d \Omega
\end{aligned}
$$

where $C_{4}$ is a positive constant independent of $A>N_{0}$ and $m \geqq 1$. Thus we have the inequality

$$
\begin{aligned}
& \int_{S_{A}} X^{l}\left\{\gamma v_{X}^{2}-\frac{|\nabla v|^{2}}{2}+\frac{(M+\lambda)}{2} v^{2}\right\} \frac{d S}{\sqrt{\gamma}} \\
& -\frac{(n-2) l+\eta}{2} \int_{S_{A}} X^{l-1} \sqrt{\gamma}\left|v v_{X}\right| d S+\frac{1}{2} \int_{\Omega_{A}} X^{l-1}\{2(l-\varepsilon)(M+\lambda) \\
& \left.+X M_{X}-m C_{4} / X^{2}\right\} v^{2} d \Omega \leqq 0
\end{aligned}
$$

for $A>N_{2}$. Using the equality

$$
|\nabla v|^{2}=X^{2 m}|\nabla u|^{2}+2 m X^{2 m-1} \gamma u u_{X}+m^{2} X^{2 m-2} \gamma u^{2},
$$

the first term of (3.12) can be written in the form

$$
\begin{gathered}
\int_{S_{A}} X^{l}\left\{\gamma v_{X}^{2}+\frac{X^{2 m}}{2}\left(M-\gamma m^{2} / X^{2}\right) u^{2}-m X^{2 m-1} \gamma u u_{X}\right\} \frac{d S}{\sqrt{\gamma}} \\
+\frac{1}{2} \int_{S_{A}} X^{2 m+l}\left(-|\nabla u|^{2}-\lambda u^{2}\right) \frac{d S}{\sqrt{\gamma}}
\end{gathered}
$$

Multiplying this by $A^{2-2 m-l}$ and integrating with respect to $A$ from $\xi$ to $\infty\left(\xi>N_{2}\right)$, we have, by Lemma 3.1 ,

$$
\begin{aligned}
& \int_{\Omega_{\xi}} X^{2-2 m} \gamma v_{X}^{2} d \Omega+\frac{1}{2} \int_{\Omega_{\xi}}\left(X^{2} M-\gamma m^{2}\right) u^{2} d \Omega+(1-m) \int_{\Omega_{\xi}} X \gamma u u_{X} d \Omega \\
& +\frac{1}{2} \int_{S_{\xi}} X^{2} \sqrt{\gamma} u u_{X} d S \\
& =\int_{\Omega_{\xi}} X^{2-2 m} \gamma v_{X}^{2} d \Omega+\frac{1}{2} \int_{\Omega_{\xi}}\left\{X^{2} M-\gamma m^{2}+(m-1)(X \gamma \sqrt{G})_{X} / \sqrt{G}\right\} u^{2} d \Omega \\
& +\frac{m-1}{2} \int_{S_{\xi}} X \sqrt{\gamma} u^{2} d S+\frac{1}{2} \int_{S_{\xi}} X^{2} \sqrt{\gamma} u u_{X} d S .
\end{aligned}
$$


Here we have used (2.6) with $\psi=X^{2}$. Thus we have from (3.12)

$$
\begin{aligned}
& \int_{\Omega_{\xi}}\left\{X^{2} M-\gamma m^{2}+(m-1)(X \gamma \sqrt{G})_{X} / \sqrt{G}-(n l-2 l+\eta)^{2} / 4\right\} u^{2} d \Omega \\
& +\int_{S_{\xi}} X^{2} \sqrt{\gamma} u u_{X} d S+(m-1) \int_{S_{\xi}} X \sqrt{\gamma} u^{2} d S \\
& +\int_{\xi}^{\infty} A^{2-2 m-l} d A \int_{\Omega_{\Delta}} X^{l-1}\left\{2(l-\eta)(M+\lambda)+X M_{X}-m C_{4} / X^{2}\right\} v^{2} d \Omega \\
& \leqq 0
\end{aligned}
$$

for $\xi>N_{2}$. (Note that $\{(n-2) l+\eta\} X^{1-2 m}\left|v v_{X}\right| \leq X^{2-2 m} v_{X}^{2}+\frac{1}{4}\{(n-2) l$ $\left.+\eta\}^{2} X^{-2 m} v^{2}\right)$.

Put

$$
\Phi(\xi)=\int_{S_{\xi}} X^{2} \sqrt{\gamma} u^{2} d S
$$

Then

$$
\frac{1}{2} \frac{d \Phi}{d \xi}=\int_{S_{\xi}}\left\{X^{2} \sqrt{\gamma} u u_{X}+\left(X^{2} \gamma \sqrt{G}\right)_{X} u^{2} \sqrt{\gamma} G\right\} d S
$$

By Lemma 2.2, we can choose $C_{5}>0$ such that $\left(\gamma X^{2} \sqrt{G_{X}} / \sqrt{G}\right)<\left(C_{5}-1\right) \gamma X$ for $X>N_{0}$. (3.13) and (3.14) give

$$
\begin{aligned}
& \int_{\Omega_{\xi}}\left\{X^{2} M-\gamma m^{2}+(m-1)(X \gamma \sqrt{G})_{X} / \sqrt{G}-(n l-2 l+\eta)^{2} / 4\right\} u^{2} d \Omega \\
& +\frac{1}{2} \frac{d \Phi}{d \xi}+\left(m-C_{5}\right) \frac{1}{\xi} \Phi \\
& +\int_{\xi}^{\infty} A^{2-2 m-l} d A \int_{\Omega_{A}} X^{l-1}\left\{2(l-\eta)(M+\lambda)+X M_{X}-m C_{4} / X^{2}\right\} v^{2} d \Omega \\
& \leqq 0 .
\end{aligned}
$$

The coefficient of $u^{2}$ in the first integral of (3.15) tends to $2 m-1-(n$ $-1) l-(n l-2 l+\eta)^{2} / 4$ as $X \rightarrow \infty$. See Lemmas 2.1, 2.2 and 2.3. So it is positive if $m>C_{6}>\{1+(n-1) l\} / 2+(n l-2 l+\eta)^{2} / 8$ and $X$ is sufficiently large, say $X>N_{5}$. We can take $N_{5}$ independently of $m$, at least, when $m>C_{6}$. If we put 


$$
\begin{aligned}
& 2(l-\eta)(M+\lambda)+X M_{X}-\frac{C_{4}}{X^{2}} \\
& =2(l-\eta-1) m^{2} / X^{2}+2(l-\eta) \lambda-m h,
\end{aligned}
$$

then, by Lemma 2.3, we can take a positive constant $C_{7}$ such that $|h|<$ $C_{7} / X^{2}$ for $X>N_{0}$. Now if we put in (3.15) and (3.16) $m=m(\xi, \eta)=$ $\xi \sqrt{\lambda(l-\eta) /\left(1-l+\frac{3}{2} \eta\right)}$, then there exists positive $N_{6}(\eta)$ such that $m>$ $C_{6}, m>C_{7} / \eta$ for $\xi>N_{6}$. Note that

$$
\begin{aligned}
& (3.16)=2(l-\eta-1)(l-\eta) \lambda \xi^{2} /\left(1-l+\frac{3}{2} \eta\right) X^{2}+2(l-\eta) \lambda-m h \\
& \geqq 2 \lambda(l-\eta)\left(1-\frac{\xi^{2}}{X^{2}}\right)+\left(m \eta-C_{7}\right) m / X^{2} \\
& >0
\end{aligned}
$$

if $X>\xi>N_{6}$. Taking $\eta$ sufficiently small we may assume $m(\xi, \eta) / \xi>\alpha$. Moreover, for such $\eta$, we can take $N_{7}(\eta)\left(>N_{6}(\eta)\right)$ so that $\left(m(\xi, \eta)-C_{5}\right)$ $\mid \xi>\alpha$ for $\xi>N_{7}$. Thus we have from (3.15) the differential inequality

$$
\frac{d \Phi}{d \xi}+2 \alpha \Phi \leqq 0
$$

for large $\xi$. This proves the lemma.

Q.E.D.

Lemma 3.3. Under the assumption of Lemma 3.1,

$$
\int_{\Omega_{1}(N)} e^{2 \alpha X}\left(u^{2}+|\nabla u|^{2}\right) d \Omega<\infty
$$

for $\alpha<\sqrt{\lambda l /(1-l)}$. If $l=1, \alpha$ may be taken arbitrarily.

Proof. We assume that $u$ is real valued. Replace $\psi$ in (2.6) by $e^{2 \alpha X}$, then we have

$$
\begin{aligned}
& \int_{\Omega_{A B}} e^{2 \alpha X}|\nabla u|^{2} d \Omega=\left\{\int_{S_{A}}-\int_{S_{B}}\right\} \sqrt{\gamma} e^{2 \alpha X} u u_{X} d S \\
& -2 \alpha \int_{\Omega_{A B}} \gamma e^{2 \alpha X} u u_{X} d \Omega+\lambda \int_{\Omega_{A B}} e^{2 \alpha X} u^{2} d \Omega .
\end{aligned}
$$


Next note that

$$
\begin{aligned}
& \int_{\Omega_{A B}} \gamma e^{2 \alpha X} u u_{X} d \Omega=-\frac{1}{2} \int_{\Omega_{A B}}\left(\sqrt{\gamma} e^{2 \alpha X}\right)_{X} \sqrt{\gamma} u^{2} d \Omega \\
& +\frac{1}{2}\left\{\int_{S_{B}}-\int_{S_{A}}\right\} \sqrt{\gamma} e^{2 \alpha X} u^{2} d S .
\end{aligned}
$$

In view of Lemma 3.2 , the limit of (3.19) exists when $B \rightarrow \infty$. Hence

$$
\lim _{B \rightarrow \infty} \inf \left|\int_{S_{B}} \sqrt{\gamma} e^{2 \alpha X} u u_{X} d S\right|=0
$$

Thus the limit of (3.18) exists when $B \rightarrow \infty$.

Q.E.D.

\section{Proof of Theorem 1.1}

If the assertion of Theorem 1.1 is not true, there exists some $\delta>0$, and

$$
\underset{t \rightarrow \infty}{\lim \inf } t^{\delta} \int_{P_{t}}\left(|u|^{2}+|\nabla u|^{2}\right) d S=0
$$

This is nothing but (3.1) of Lemma 3.1. Thus, for the proof of Theorem 1.1, it suffices to show the following assertion.

Let $u$ be a solution of (1.1) and (1.2). If $u$ satisfies (3.1), then $u \equiv$ 0 on the whole of $\Omega$.

First note that

$$
\int_{\Omega_{1}(N)} X^{k} e^{m X^{\beta}}\left(|u|^{2}+|\nabla u|^{2}\right) d \Omega<\infty
$$

for any $m>0, k>0$ and $\beta<1$. This is a direct consequence of Lemma 3.3 .

Put $v=e^{m X^{\beta}} u$, then

$$
\Delta v-2 m \beta X^{\beta-1} \gamma v_{X}+(L+\lambda) v=0,
$$

where 


$$
L=m^{2} \beta^{2} X^{2 \beta-2}-m \beta(\beta-1) X^{\beta-2}-m \beta X^{\beta-1}(\gamma \sqrt{G})_{X} / \sqrt{G} .
$$

We multiply (4.2) by $X^{k} v_{X}$ and integrate over $\Omega_{A}$. From (4.1) we have

$$
\begin{aligned}
& -\int_{S_{A}} X^{k}\left\{r v_{X}^{2}-\frac{|\nabla v|^{2}}{2}+\frac{(L+\lambda)}{2} v^{2}\right\} \frac{d S}{\sqrt{r}} \\
& -\int_{\Omega_{A}}\left(2 m \beta X^{\beta+k-1}+(k-l) X^{k-1}\right) \gamma v_{X}^{2} d \Omega+ \\
& \frac{1}{2} \int_{\Omega_{A}} X^{k-1}\left[(k+(n-3) l+\eta)|\nabla v|^{2}-\{(k+(n-1) l-\eta)(L+\lambda)\right. \\
& \left.\left.+X L_{X}\right\} v^{2}\right] d \Omega \\
& \geqq 0
\end{aligned}
$$

for $\eta>0$ and sufficiently large $A$, say $A>N_{8}(\eta)$. (See the proof of Lemma 2.5.) If we put $k=(3-n) l-\eta$ in (4.4),

$$
\begin{aligned}
& \int_{S_{A}} X^{(3-n) l-\eta}\left\{\gamma v_{X}^{2}-\frac{|\nabla v|^{2}}{2}+\frac{(L+\lambda)}{2} v^{2}\right\} \frac{d S}{\sqrt{\gamma}} \\
& \leqq-\int_{\Omega_{A}}\left\{2 m \beta X^{\beta+(3-n) l-\eta-1}+((2-n) l-\eta) X^{(3-n) l-\eta-1}\right\} \gamma v_{X}^{2} d \Omega \\
& -\int_{\Omega_{A}} X^{(3-n) l-\eta-1}\left\{(l-\eta)(L+\lambda)+X L_{X}\right\} v^{2} d \Omega=I_{1}+I_{2} .
\end{aligned}
$$

There is $N_{9}(\eta)\left(>N_{8}(\eta)\right)$ such that $I_{1} \leqq 0$ for $A>N_{9}$. Assuming $m \geqq 1$, and $\frac{1}{2}<\beta<1$, we can take $N_{9}$ independently of $m$ and $\beta$.

Next note that

$$
\begin{aligned}
& L \geqq m^{2} \beta^{2} X^{2 \beta-2}-m C_{8}(\beta) X^{\beta-2} \\
& X L_{X} \leqq m^{2} \beta^{2}(2 \beta-2) X^{2 \beta-2}+m C_{9}(\beta) X^{\beta-2},
\end{aligned}
$$

where $C_{8}(\beta)$ and $C_{9}(\beta)$ are constants which are independent of $m$.

Now let us assume $\eta$ is small so that $l-\eta>0$. If we take $\beta(<1)$ near to 1 , then $l-\eta>2(1-\beta)$, and hence

$$
(l-\eta)(L+\lambda)+X L_{X}
$$




$$
\begin{aligned}
& \geqq m^{2} \beta^{2}(l-\eta+2 \beta-2) X^{2 \beta-2}-m\left\{(l-\eta) C_{8}(\beta)-C_{9}(\beta)\right\} X^{\beta-2} \\
& \geqq 0
\end{aligned}
$$

for large $m$ and $X$, say $m \geq C_{10}$ and $X \geq N_{10}(\eta)\left(>N_{9}\right)$, where $N_{10}$ is independent of $m\left(>C_{10}\right)$. Thus we have from (4.5)

$$
\int_{S_{A}} X^{(3-n) l-\eta}\left\{\gamma v_{X}^{2}-\frac{|\nabla v|^{2}}{2}+\frac{(L+\lambda)}{2} v^{2}\right\} \frac{d S}{\sqrt{\gamma}} \leqq I_{1}+I_{2} \leqq 0
$$

for $A>N_{10}$ and $m>C_{10}$.

On the other hand, if we put

$$
\begin{aligned}
& \int_{S_{A}} X^{(3-n) l-\eta}\left\{\gamma v_{X}^{2}-\frac{|\nabla v|^{2}}{2}+\frac{(L+\lambda)}{2} v^{2}\right\} \frac{d S}{\sqrt{\gamma}} \\
& =m^{2} M_{1}(u, A)+m M_{2}(u, A)+M_{3}(u, A),
\end{aligned}
$$

(where $M_{1}, M_{2}$ and $M_{3}$ are independent of $m$,) then it is easy to see $M_{1}(u, A)>0$ when $u \neq 0$ on $S_{A^{*}}$. Note that $v=e^{m X^{\beta}} u$. If we assume $M_{1}(u, A)>0$ for some $A>N_{10}$,

$$
\int_{S_{A}} X^{(3-n) l-\eta}\left\{\nu v_{X}^{2}-\frac{|\nabla v|^{2}}{2}+\frac{(L+\lambda)}{2} v^{2}\right\} \frac{d S}{\sqrt{\gamma}}>0
$$

for sufficiently large $m$. This contradicts (4.6), hence we see $u \equiv 0$ on $\Omega_{N_{10}}$. The unique continuation theorem for the second order elliptic equations enables us to conclude $u \equiv 0$ on the whole of $\Omega$. The proof of Theorem 1.1 is now complete.

\section{On the Spectrum of $-\Delta$}

This final brief section concerns the spectrum of $-\Delta$ in $\Omega$ with the Dirichlet boundary condition.

Let $L$ be the operator in $L^{2}(\Omega)$ with the domain $D(L)=\left\{f: f \in D_{L^{2}}^{1}\right.$, $\left.\Delta f \in L^{2}(\Omega)\right\}$, and $L u=-\Delta u$, where $D_{L^{2}}^{1}$ is the completion of $C_{0}^{\infty}(\Omega)$ with regard to the norm

$$
\|f\|_{1}=\left\{\int_{\Omega}\left(|f|^{2}+|\nabla f|^{2}\right) d \Omega\right\}^{\frac{1}{2}}
$$


Then $L$ is a non-negative self-adjoint operator in $L^{2}(\Omega)$.

Theorem 5.1. Under the assumption on $\Omega$ in $\$ 1, L$ has no point eigenvalues. Moreover, the continuous spectrum of $L$ fills up the non-negative half of the real axis.

Proof. $L$ is a non-negative operator, and so it has no negative eigenvalues. Let $u \in D(L)$, and $L u=0$. Integrate $u L \bar{u}$ over $\Omega$, we have

$$
\int_{\Omega}|\nabla u|^{2} d \Omega=0
$$

Hence $u=$ constant. By the Diriclet condition, $u=0$, and so $\lambda=0$ cannot be an eigenvalue of $L$. If $u \in D(L)$, then

$$
\liminf _{t \rightarrow 0} t \int_{P_{t}}\left(|u|^{2}+|\nabla u|^{2}\right) d S=0
$$

This shows that the non-existence of positive eigenvalues is a consequence of Theorem 1.1.

Next let us prove the latter half of the theorem. That is, we must prove that any non-negative real number $\lambda$ belongs to the spectrum of $L$.

Let $\varphi=\varphi\left(X_{1}, \ldots, X_{n}\right)$ be a function which is in $C_{0}^{\infty}\left(\Omega_{N_{0} N_{0}+1}\right)$, and $\varphi_{m}=\varphi\left(X_{1} / m, X_{2}, \cdots, X_{n}\right)$. Put

$$
\Phi_{m}=e^{i \sqrt{\lambda} X_{1}} \varphi_{m} / \nu_{m},
$$

where $\nu_{m}=\left\|\varphi_{m}\right\|_{L^{2}(\Omega)}$. It is not difficult to show that

$$
\left\|L \Phi_{m}-\lambda \Phi_{m}\right\|_{L^{2}(\Omega)} \rightarrow 0 \quad(m \rightarrow \infty) .
$$

Taking subsequence if necessary, we may assume supp $\Phi_{\text {in }} \operatorname{supp} \Phi_{j}=\phi(i \neq j)$ $\left(\Phi_{i}\right.$ and $\Phi_{j}(i \neq j)$ are orthogonal), where supp $\Phi$ denotes the support of $\Phi$. This shows that $\lambda$ is in the spectrum of $L$, because, if not, $\left\{\Phi_{m}\right\}$ would tend to 0 , which is impossible, however, on account of $\left\|\Phi_{m}\right\|_{L^{2}(\Omega)}=1$.

Q.E.D. 


\section{References}

[1] Rellich, F., Über das asymptotische Verhalten der Lösungen von $(\Delta+\lambda) u=0$ in unendlichen Gebieten, Jber. Deutsch. Math. Verein. 53 (1943), 57-65.

[2] Jones, D. S., The eigenvalues of $\Delta^{2} u+\lambda u=0$ when the boundary conditions are given on semi-infinite domains, Proc. Cambridge Philos. Soc. 49 (1953), 668-684.

[3] Agmon, S., Lower bounds for solutions of Schrödinger type equations in unbounded domains, Proc. International Conference on Functional Analysis and Related Topics, Tokyo 1969, 216-230.

[4] Roze, S. N., On the spectrum of a second order differential operator, Mat. Sb. 80 (1969), 195-209.

[5] Eidus, D. M., The principle of limit amplitude, Russian Math. Surveys, 24 (1969), 97-157. 
